\title{
Temperature Spectra from a Turbulent Free Thermal Plume and in Interaction with its Material Environment
}

\author{
J. Zinoubi ${ }^{1 \dagger}$, T. Naffouti ${ }^{2}$ and R. B. Maad $^{2}$ \\ ${ }^{1}$ Institut Préparatoire aux Etudes d'Ingénieurs El-Manar, El-Manar 2092, Tunis, Tunisie. \\ ${ }^{2}$ Faculté des Sciences de Tunis, Département de Physique, Laboratoire d'Energétique et des Transferts Thermique et \\ Massique, El-Manar 2092, Tunis, Tunisie \\ †Corresponding Author Email: jamil_zinoubi@yahoo.fr
}

(Received July 6, 2009; accepted March 25, 2010)

\begin{abstract}
In this work we study the interaction of an axysymmetric thermal plume with a thermosiphon flow that surrounds it. The thermal plume is created by a circular disk heated by joule effect at constant temperature. The disk is placed at an open ended vertical cylinder on a quiet constant temperature. The internal wall of the cylinder heats up under the effect of thermal radiation emitted by the hot source. The confinement of the fluid causes, in the bottom part of the cylinder, an aspiration of the fresh air. It is a thermosiphon flow that comes to interact with the plume. By studying the average and fluctuating thermal fields it was found that the flow of the plume is strongly influenced by the presence of nearby walls. It was noted that the vertical transport becomes more intense and the structure of the flow becomes more turbulent. On the other hand, we attend a fast homogenization of the flow in the upper cylinder. To obtain more detailed information of this flow, we develops, during this study, a spectral analysis of the fluctuating thermal fields for the case of a plume evolving in unlimited and in an enclosed environment. The energy spectra study shows an important shift of the energy peaks toward the high frequencies under the effect of the thermosiphon. As destroying structures them on a big scale generated by the plume, the thermosiphon provokes a fast mixture of the fluid thus while leading to vortex of weaker size.
\end{abstract}

Keywords: Axysymmetric thermal plume, Turbulent structure, Confinement, Power spectral density, Cascade concept of Kolmogorov.

\section{NOMENCLATURE}

$\mathrm{D}$

$\mathrm{E}_{\mathrm{T}} \quad$ Power spectral density of the

Diameter of the disk heated source,

$m$ temperature,

$$
E_{T}(f)=\int_{-\infty}^{+\infty} R_{T}(\tau) e^{-j 2 \pi f t} d t
$$

f Frequency, $\mathrm{Hz}$

$\mathrm{I}_{\mathrm{t}}$

$r$

$\mathrm{R}_{\mathrm{T}}(\tau)$
$R$
$\mathrm{~T}$
$\mathrm{~T}$

$\mathrm{~T}_{\mathrm{a}}$
$\mathrm{T}_{\mathrm{S}}$
$\mathrm{Z}$

auto-correlation function,

$$
\left(=\frac{\overline{T^{\prime}(t) T^{\prime}(t+\tau)}}{\overline{T^{\prime 2}(t)}}\right)
$$

Radius of the disk heated source, $m$ Average temperature, ${ }^{\circ} \mathrm{C}$ Instantaneous temperature fluctuation, ${ }^{\circ} \mathrm{C}$ Temperature of the ambient air, ${ }^{\circ} \mathrm{C}$ Temperature of the hot source, ${ }^{\circ} \mathrm{C}$ Vertical coordinate, $\mathrm{m}$

\section{INTRODUCTION}

The study presented in this work is a contribution to understanding the turbulent thermal plume structure evolving in a free and in a confined environment. This type of flow results from an initial temperature field (generated by the presence of a hot source), that causes by the difference in density with the immediate environment where the temperature is lower, the moving air particles initiating the plume. This kind of the flow occurs in many forms in nature and in

industrial environments such as, we can mention for example, fires in forests and in buildings, the discharge of hot air and smoke from industrial chimneys, , where the plumes are composed of hot air and combustion products (toxic gases), constitute ecological dangers. At smaller scales, the release of heat from electronic components can cause similar flows. The study of fundamental properties of the turbulent flows of natural convection should contribute to a better understanding of convective complex phenomena that accompany it. Knowledge of the physical mechanisms governing the 
development of these flows would permit to better control their evolution and design, followed by the appropriate methods of prevention and safety against the dangers of such flows.

Most previous investigators have focused their attentions on the structure of a thermal plume evolving in a free and unlimited environment driven primarily by buoyancy forces. This type of flow has been a subject of several theoretical and experimental studies (Agator and Doan Kim, 1982; Brahimi and Doan Kim, 1986; Dehmani and Doan Kim, 1996a, 1996b; Dai et al, 1994, 1995; Elicer-Cortés et al., 2000, 2004, 2006; Fukui Keisuke et al, 1991; Fulachier, and Antonia, 1984; Noto and Okamoto, 1990-1991, Shabbir and George, 1994; Sangras et al. 1998; Takeo et al, 2004; Minh Vuong and Frédéric, 2006).

This situation, (the evolution in a free and unlimited environment), is difficult to meet, and generally the plume is moving in an environment limited by walls that can affect the flow. It is therefore very important to determine the effect of the nearby wall on the flow development mechanisms.

The thermal plume interacts closely with its physical environment and the structure of the resulting flow becomes a lot more complicated. Indeed, the plume flow in confined environments is not independent of that along the adjacent walls (thermosiphon flow). The two flows are coupled in most cases. This interaction introduces considerable complexity in the analysis of internal flows. The interaction problem was not addressed. The only work to our knowledge in this thematic are: the effect of a vertical surface adjacent on the flow plume (Agator, 1983) the interaction between two equal thermal plumes and 'unequal power (Agator, 1983; Brahimi 1988, 1989; Pera and Gebhart, 1975) and the interaction of the plume with a thermosiphon (Mahmoud et al, 1998; Zinoubi et al, 2003, 2004; Bouslimi and Dehmani, 2005; Abdelhakim and René, 2007).

The turbulent structure can be considered as elements vortex stretching each other. A longer vortex is an essential aspect of turbulent motion. It produces the passage of the energy to more and more small scales until the viscous forces become active and dissipate energy.

The flow studied is subject to many vortex movements whose the precise knowledge is needed for an appropriate description of the flow general characteristics. This determination of the turbulence fine structure, particularly at the size and the frequency of the vortex containing the most energy, can be accomplished only by using spectral analysis. To determine the spectral characteristics of indiscriminate temperature signals, we used the Fast Fourier Transform «FFT: Fast Fourier Transform ».

Thus we were led to develop in this article the study of the plume characteristics from its source until its interaction with its physical environment.

\section{EXPERIMENTAL APPARATUS AND MEASUREMENT TECHNIQUES}

The thermal plume is created by a flat disk having a $0.07 \mathrm{~m}$ diameter (Fig.1a). The plume source is electrically heated at $300^{\circ} \mathrm{C}$ on the surface by Joule effect. The disk is placed inside the cylinder, at $5 \mathrm{~cm}$ of the entrance of the open ended steel vertical cylinder (Fig.1b), which has a $0.15 \mathrm{~m}$ diameter and a $0.5 \mathrm{~m}$ height. The system is placed on a frame at $80 \mathrm{~cm}$ above the ground to allow air supply from below. The uniformity of the source surface temperature is obtained by the use of wire resistors mounted behind the disk. A thermal regulation apparatus kept the temperature of the disk as uniform as possible within a good approximation. $\mathrm{Al}-\mathrm{Cr}$ thermocouples are used to measure the surface temperature of the disk. The difference in temperature between the end and the centre of the disk is less than $5^{\circ} \mathrm{C}$. The vertical cylinder was thermally insulated by an Armaflex cylindrical bed, which has a $0.02 \mathrm{~m}$ thickness.

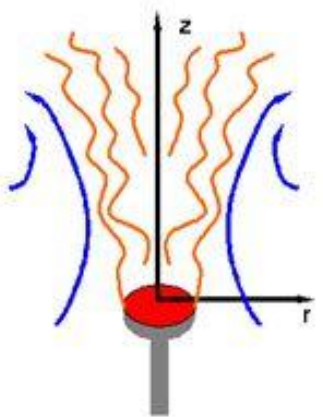

(a)

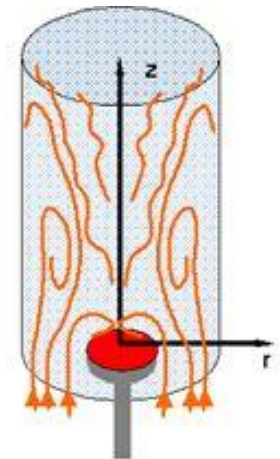

(b)
Fig. 1. Experimental configuration (a): Free plume, (b): Plume in interaction

The strong dependence of the flow to the surrounding conditions, require making evolve him inside a quiet atmosphere; so, the experimental device has been placed in an independent closed room.

To explore the voltage signals corresponding to the temperature we have used the resistant wire anemometer at constant current. This technique adopted for a long time in natural convection, is based on the principle of the resistance variation of a platinum wire (7.5 $\mu \mathrm{m}$ in diameter).

In order to avoid the disruption of the flow, the probe is introduced vertically, by the system exit, so that its sensible wire was perpendicular to the ascending flow. The wire thermal inertia, where its time constant is the order of $1 \mathrm{~ms}$, introduced not of measure mistake, especially to the weak frequencies met in plumes (Doan Kim, 1977; Brahimi, 1989; Zinoubi, 2003;). Mistakes coming from the probe calibration are lower than $1 \%$.

On the other hand, in order to explore thermal voltage signals at every level of the flow, we have used a displacement system of the probe, piloted by a computer, following two directions. The minimal displacement in the vertical direction is $10^{-3} \mathrm{~m}$, whereas in the horizontal direction is $210^{-5} \mathrm{~m}$. 
A computer provided of a data acquisition card permits to appropriate of the instantaneous measures all $10 \mathrm{~ms}$ and to record signals for further statistic processing.

\section{METHODOLOGY FOR OBTAINING POWER SPECTRA}

A random process $\mathrm{x}(\mathrm{t})$ is represented by a phenomenon where the variable may take several possible values with a probability distribution.

These random processes are studied in the temporal space, but it is more convenient to study in the frequency space, because the most frequently, we search the energy density or power density according to the frequency values. In other words, the term "spectral" shows that the domain studied is the frequency.

\subsection{Fourier Transform of Temperature Fluctuant Field}

The physical operation of the Fourier transform (Fourier 1822) is a representation of the fluctuating field as a random superposition of undulating movements characterized by a wave vector $\overrightarrow{\mathrm{K}}$ and a corresponding spectral density En. The $\overrightarrow{\mathrm{K}}$ orientation represents the evolution direction of turbulent structures, while the $\mathrm{K}$ module or the wave number is related to the size of these structures (inverse of the size of the vortices).

\subsection{Techniques of the Spectral Analysis}

For a long time, the study of spectral analysis is performed mainly with the spectrum analyzers that are based on the principle of frequency filtering.

In recent years, some researchers have tried several types of analyzers, each with advantages and disadvantages.

- The analyzer point by point is accurate but needs to work offline because of the treatment duration.

- The analyzer of battery of selective filter operates in real time but it is very difficult to achieve.

- The analyzer of time compression also operates in real time but the band treated is limited. On the other hand, it was found that all these analyzers have in common other defects: the need to double them when we want to measure cross-spectra.

Later, the trend is oriented towards the use of digital signal processing. These methods make use of the Fourier transform.

For the Fourier transformation in question, the calculation is very long, was a serious obstacle to the spectra study. In 1965, J. W. COOLEY and J. W. TUKEY proposed a simple algorithm that reduces very considerably the computation time. This method is the Fast Fourier Transform.

In this work we used the fast Fourier transform to perform spectral analysis of temperature fluctuations after making the auto-correlation. The Fourier transform of the latter is the spectral power density of the signal.

\section{Main ReSUlts}

\subsection{Means and Fluctuating Thermal Fields: Global View}

In Figs. 2 and 3 are presented the isotherms plotted in a symmetry plane of the flow, for both configurations studied. These isotherms show an overall picture of the thermal field giving information on the region occupied by the plume during its ascent. These isotherms can also rediscover the different zones of the flow previously reported. For the case of a free plume, Fig. 2 shows that the isolated plume supply themselves regularly in fresh air by the low and sides shows the entrainment phenomenon of fresh air from the surroundings ambient air by the plume. This figure shows just above the source, the strong thermal gradients due to a brutal transformation of the ambient air in hot plume. Away from the source, a lateral extension of the plume is observed accompanied by a decrease in temperature gradients until the extinction of the plume

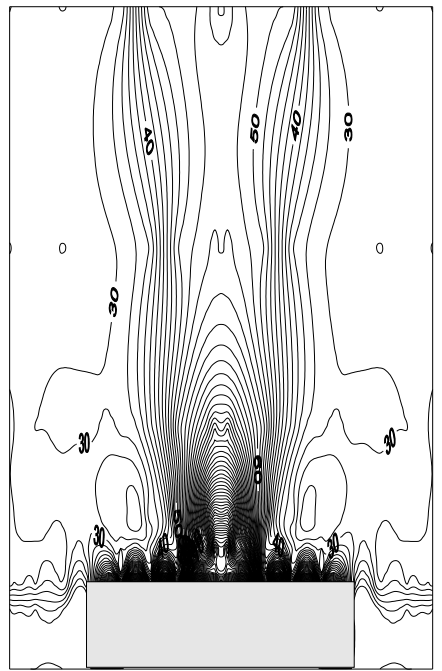

Fig. 2. Thermal average field: Free plume

In the case of confined plume, Figure 3 shows that the isothermal lines undergo profound changes in forms and values compared to those isolated plume. This figure shows that the plume and the cylinder wall supply themselves regularly in fresh air by the low. It also shows that the flow is divided in three zones: a first zone characterized by strong temperature gradients followed by a contraction of flow. Away from the source we noted that the plume extends toward the cylinder wall. At this level, the cylinder wall acts as a barrier before this expansion, there is a blockage of the ascending flow. Beyond this level, a spacing of the isothermal lines is observed, leading to very low gradients of temperature.

Figures 4 and 5 show the iso-values of the thermal turbulence rate plotted in a symmetry plane of the flow, for both configurations studied, these iso-values informs us about the more or less turbulent flow region. Indeed, in the case of a free plume, Figure 4 shows a tightening of the iso-values lines in the vicinity of the source, in the radial direction. This is due to strong gradients of the turbulence rate in this zone. It also 
shows an important line spacing in the axial direction with low gradients of turbulence rate. These low gradients are due to the lateral expansion of the plume.

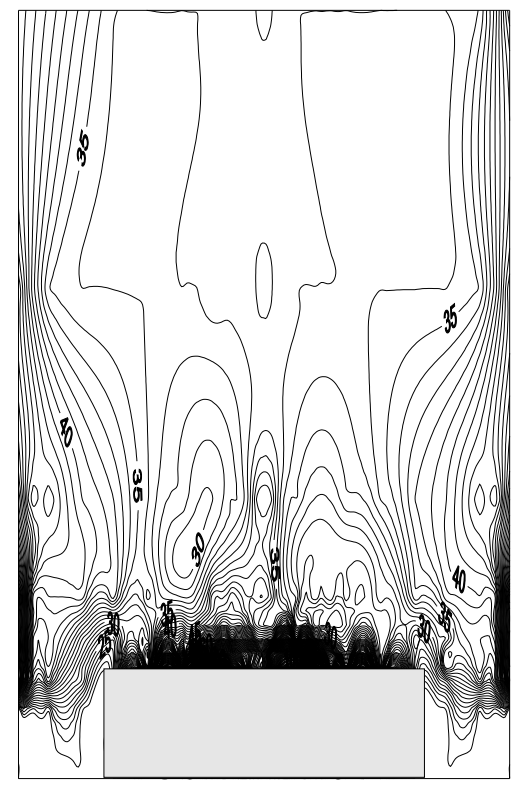

Fig. 3. Thermal average field: Plume in interaction

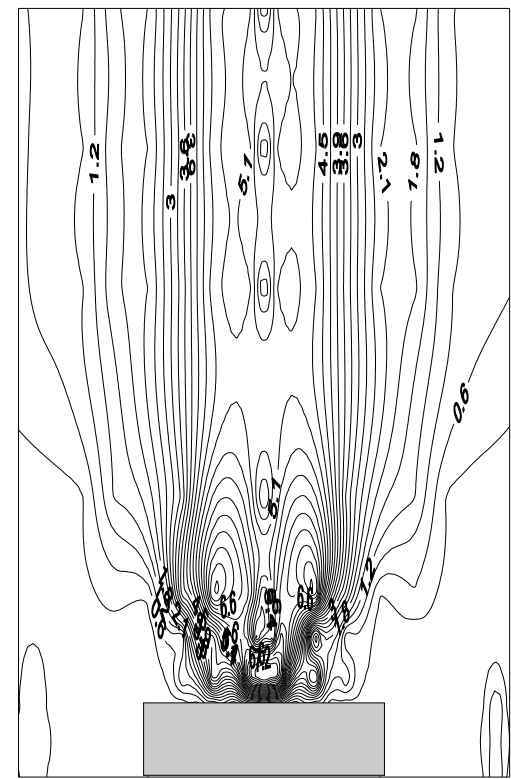

Fig.4. Iso-values of the thermal turbulent rate: Free plume

In the case of confined plume, Fig. 5 shows that the confinement of the plume considerably affects its structure. Indeed, just above the source, a tightening of the iso-values lines of the thermal turbulence rate is observed with a rotating vortex structure. Away from the source, we notice line spacing due to a homogenization of the flow

\subsection{Spectral Analysis of Temperature Fluctuating}

The spectral energy density is designated by $E_{T}(f)$. The input signal is processed in accordance with the
Shannon's theorem $(1 / \mathrm{Te}>2 \mathrm{fM})$, i.e. the study of 2048 signal successive slices of $\mathrm{Te}=10 \mathrm{~ms}(\mathrm{fe}=100 \mathrm{~Hz})$. The dominant frequency found in the plume $\left(\mathrm{f}<\mathrm{f}_{\mathrm{M}}=25 \mathrm{~Hz}\right)$

We will examine the various spectra of temperature fluctuations for several positions of the probe. Because of the similarity between the spectra recorded in each zone of the flow, we are constrained to present the spectra on three study sections $(\mathrm{z} / \mathrm{D}=0285,0714$ and 4.28) for both configurations studied: isolated plume and plume in interaction.

\subsubsection{Plume Evolving in a Free and Unlimited Environment: Isolated Plume}

In this study, the treatment of spectra of temperature fluctuations is performed using the fast Fourier transform of the auto-correlation of signals delivered by the probe.

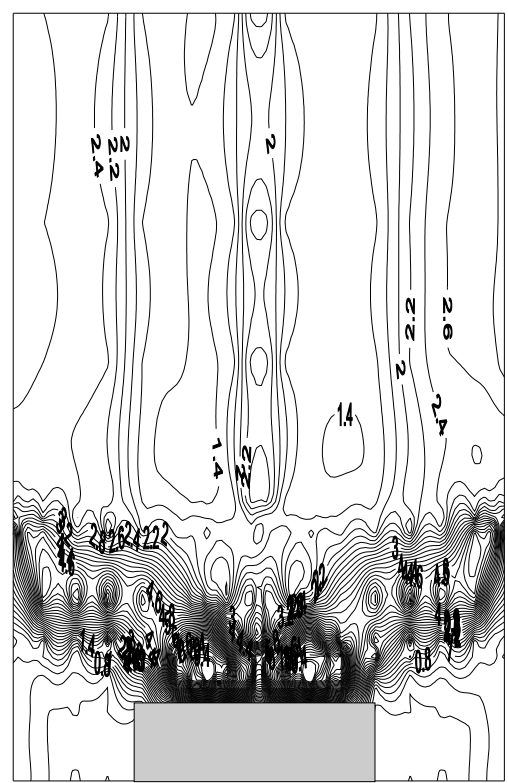

Fig. 5. Iso-values of the thermal turbulent rate: Plume in interaction

We present in Fig. 6 the temperature spectra for the levels $(\mathrm{z} / \mathrm{D}=0285,0714$ and 4.28) and for three positions of the probe.

For the $\mathrm{z} / \mathrm{D}=0.285$ Fig. $6 \mathrm{a}$ shows the spectra for different radius. Indeed, on the plume axis $(r / R=0)$, mid-radius $(r / R=0.5)$ and the source border $(r / R=1)$ we notice the existence of the most important peak at the frequency $0.1 \mathrm{~Hz}$. This peak is characteristic the big vortex energy carriers who occur from the hot source. This figure also shows the appearance near the peak at $0.1 \mathrm{~Hz}$, secondary lower peaks, as one comes closer of the source border. These peaks are related to puffs fresh air coming from the low that comes supplied the plume source.

For the level $\mathrm{z} / \mathrm{D}=0.714$ Fig. $6 \mathrm{~b}$ shows, on the plume axis $(r / R=0)$, a peak at $0.1 \mathrm{~Hz}$ with another peak at 0.8 $\mathrm{Hz}$ This peak is bound to the puff fresh air that comes to supply the hot source which is driven by the upward flow of the plume. Moreover, in the plume region where the turbulence intensity is maximal, and at the 
border of the source, this figure shows the existence of a single peak at $0.1 \mathrm{~Hz}$.This behavior reflects the laminar nature of the flow.
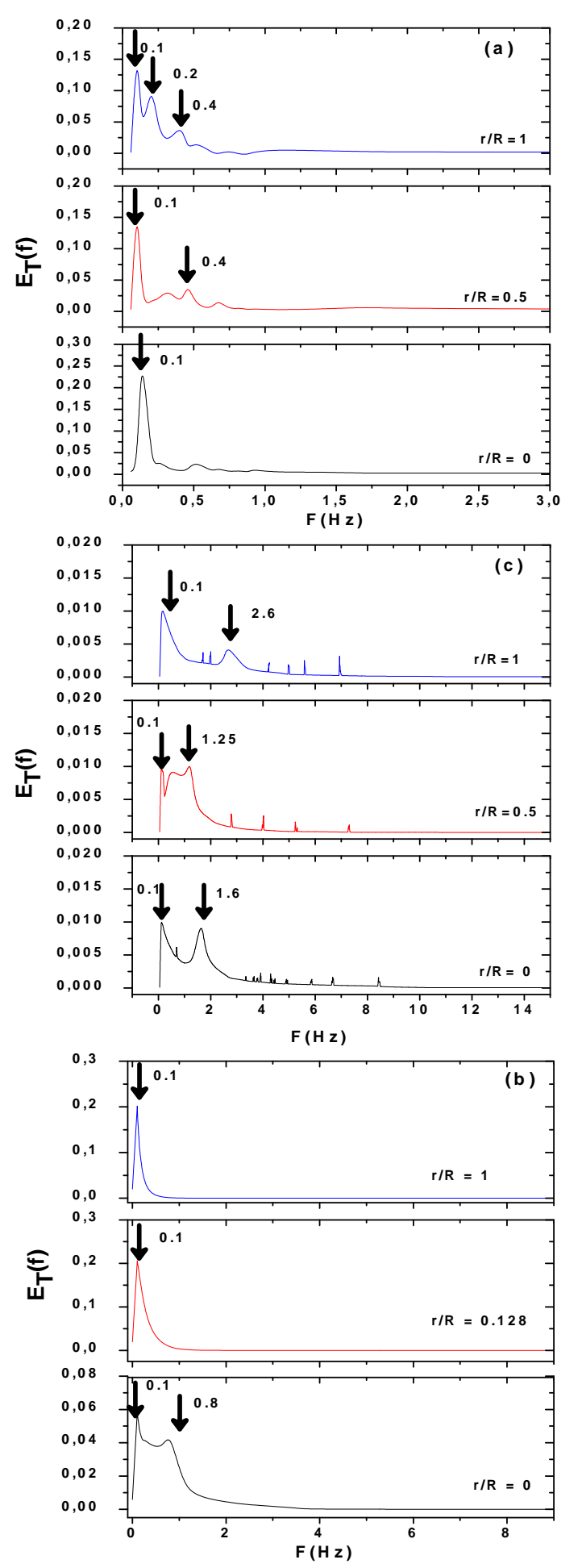

Fig. 6. Power spectral density of the temperature: Free plume: $\mathrm{a}: \mathrm{z} / \mathrm{D}=0.285, \mathrm{~b}: \mathrm{z} / \mathrm{D}=0.714$,

$$
\mathrm{c}: \mathrm{z} / \mathrm{D}=4.28
$$

In the established turbulence zone ( $\mathrm{z} / \mathrm{D}=4.28)$, Fig. $6 \mathrm{c}$ shows again the existence of the largest peak at $0.1 \mathrm{~Hz}$. This shows that the source continues to exert its major effect in the zone where the turbulence is established.
This figure also shows the existence of several peaks corresponding to the vortex structures of different sizes. These structures characterize the mixing of the fluid which takes place in this zone.

\subsubsection{Plume evolving in a semi-enclosed environment}

The spectra of temperature fluctuations on three study sections $(\mathrm{z} / \mathrm{D}=0285,0714$ and 4.28) and four positions of the probe, are shown in Fig. 7.

For the level $z / D=0285$, Fig. 7.a shows, on the plume axis, the existence of the largest peak at frequency 0.1 $\mathrm{Hz}$, corresponding to vortices of big structures, wellcharacterized the puff fresh air that comes to supply the plume through its axis. In the region where the thermal turbulence intensity is maximal, there is the appearance of other secondary peak at a higher frequency of 1.2 $\mathrm{Hz}$. This peak characterizes the frequency of formation cycle of the rotating rolls just above the source. On the edge of the hot source, this figure shows a peak at 0.1 $\mathrm{Hz}$, reflecting the strong pull of the fresh air from the source. Near the cylinder wall, this figure shows a peak at $0.1 \mathrm{~Hz}$ related to the puff that comes to supply the cylinder wall.

For the $\mathrm{z} / \mathrm{D}=0.714$, Fig. $7 \mathrm{~b}$ shows spectra of several peaks of energy that evolve according to the probe position. Indeed, near the cylinder wall, the energy spectrum has two peaks at 0.1 and $1 \mathrm{~Hz}$. As has been shown by the visualisation (Zinoubi 2003, 2004) these peaks are related to the puffs fresh air that comes to supply the cylinder wall. At the source border $(r / R=1)$ this figure shows the existence of a spectrum with three peaks located at $0.1,1.4$ and $2.3 \mathrm{~Hz}$. These three peaks it's related respectively to the three air puffs, which confirms the observations made during the visualisation (Zinoubi 2003, 2004). In the region where thermal turbulence intensity is maximal $(\mathrm{r} / \mathrm{R}=0.5)$, this figure shows that the peak at $0.1 \mathrm{~Hz}$ is accompanied by other secondary peak at frequency $1.8 \mathrm{~Hz}$. This peak is attributed to the cycle of exhaust rotating rolls. On the plume axis, the temperature spectrum shows a single peak of $0.1 \mathrm{~Hz}$. This behaviour reflects the appearance of laminar flow on the axis. On the other hand, the energy level corresponding to the big vortex structures is still very noticeable in elevation $\mathrm{z} / \mathrm{D}=0.714$ close to the source.

For the high altitude $\mathrm{z} / \mathrm{D}=4.28$, Fig. $7 \mathrm{c}$ shows the existence of vortex structures of different sizes. It also shows a shift of peak energy to high frequencies. This shows that the energy proportion corresponding to large-scale structures decreases rapidly, while that on small dissipative structures increases. The existence of dissipative vortex leads to a uniform temperature at the cylinder exit.

The temperature spectra are also presented in a semilogarithmic coordinated system, (in abscissa the frequency $f$ and in ordered the product f.Et(f)). This representation allows the identification of spectral bands that contain significant energy, simply by taking into consideration the areas delimited by the spectra (Doan Kim, 1977; Agator, 1983; Brahimi, 1989). 

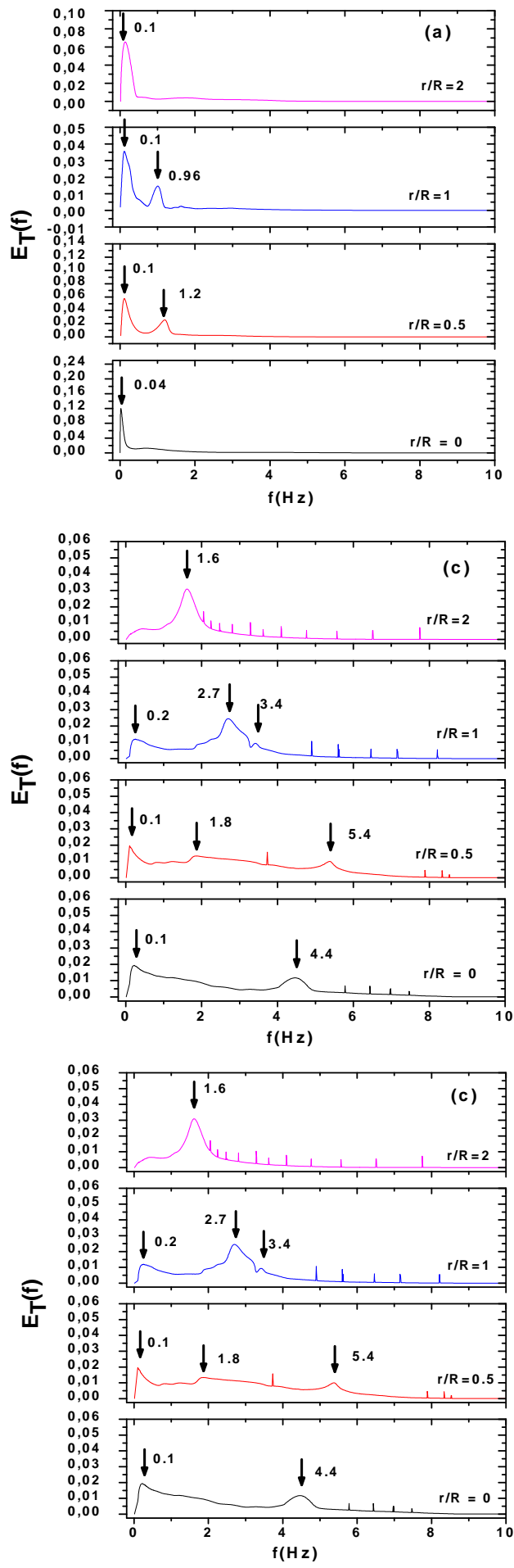

Fig.7. Power spectral density of the temperature: Plume in interaction: $\mathrm{a}: \mathrm{z} / \mathrm{D}=0.285, \mathrm{~b}: \mathrm{z} / \mathrm{D}=0.714, \mathrm{c}: \mathrm{z} / \mathrm{D}=$ 4.28

We present in Fig. 8 a comparison of energy spectra in both cases, for three positions of the probe and for the levels $\mathrm{z} / \mathrm{D}=0285,0714$ and 4.28

The comparison of temperature spectra on the two studies case shows a significant shift of peak energy to high frequencies under the of confinement effect. By destroying the vortex structures in large-scale generated by the plume, the confinement causes an increase in the number of vortex of different size and it promotes a rapid mixing of the fluid giving rise to vortex of smaller size.

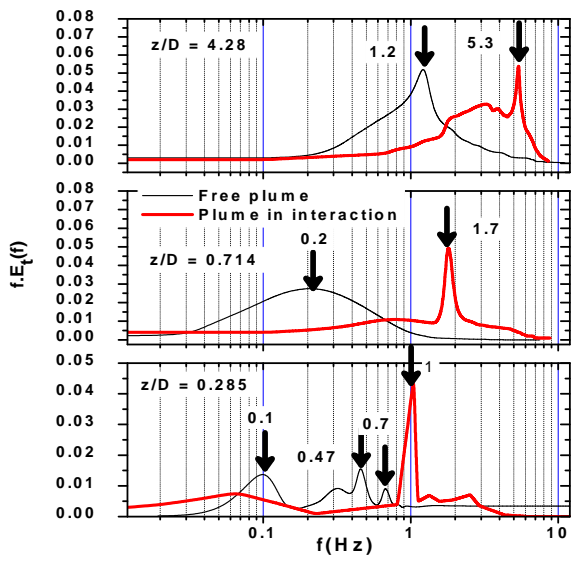

Fig. 8. Comparison of the power spectral density of the temperature in the two studied cases (Presentation semilogarithmic): $r / R=r / R\left(I_{\text {MAX }}\right)$

Figure 9 shows the vertical evolution of the spectral energy for both configurations studied. This figure shows that the energy increases with increasing altitude, and then it decreases. This behavior complies with the scenario of energy cascade toward small scales reported in previous work (Corrsin 1951; Lumley, 1965; Kotsovinos, 1991; Dai et al. 1994, 1995; Lisin and Hetsroni, 1995).

Three spectral regions have been identified, the first region is a region of production due to turbulent flow, the second region is rather interesting and is corresponding to a region of buoyancy, follows of third dissipation zone.

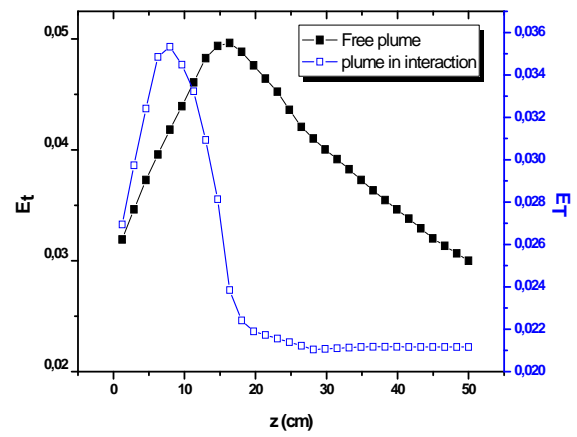

Fig. 9. Axial evolution of a total power spectral density

During the spectral analysis, we noted as one moves away of the generating plume source, changing the vortex size. Indeed, the big vortex structures, dependent on entry conditions, will produce vortices become smaller; thus transferring energy to smaller vortex. At the same time the viscosity of the fluid and the dissipation is becoming increasingly important for small vortex. Thus the inertial forces tend to expand on a range of more and more large as the viscous dissipation is not the brake. The balance between two opposing tendencies is controlled by the Reynolds 
number of turbulence. The higher the Reynolds number is high, i.e. over the viscosity is relatively small, the greater efficiency of inertial forces to transfer energy to the frequency of ever higher. During the creation of turbulence, only small frequencies are excited. The action of inertial forces is transfer energy to higher frequencies until viscous wells. There is a whole region that receives by transfer, the spectral energy. We can then assume that the small vortexes are independent of external conditions that have produced large initial vortices.

On the other hand, the vortex stretching is a key mechanism responsible for the energy cascade (Roland Schiestel, 1993). This development, due to the elongation of vortex involves the transfer of energy at scales smaller and smaller until the viscous forces become active and dissipate energy, it is the energy cascade.

In the process of energy cascade, the influence of moving average decreases for small vortexes which tend to be free of anisotropic characteristics of the largest. After several repetitions the micro-turbulence approaches isotropy (Fig. 10).

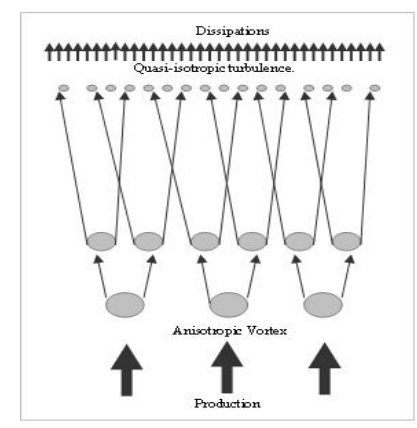

Fig.10. Bradshaw tree showing the tendency of isotropy (Roland Schiestel 1993):

(Cascade of energy)

\section{CONClusion}

During this work we studied the fine structure of a thermal plume generated by a hot disk, evolving in an unlimited and in semi confined environment. For the case of unlimited environment, the visualization of the thermal average and fluctuating field as well as the spectral study show that the global structure of the flow is divided into two separate zones; a first zone of the plume development near the source $(\mathrm{z} / \mathrm{D}=2.14)$, followed by a zone relatively remote from the hot source (z/D> 2.14), where turbulence is established. This zone is characterized by a lateral expansion of the plume. They also show the dominance of vortex structures on a big scale. The results obtained confirm the complexity of the flow due to plume-thermosiphon interaction. They showed a considerable change in the plume flow structure that develops in a semi-confined environment compared with the free flow. Indeed, the results show that the structure of the resulting flow is divided into three zones: a first zone of instability near the source $(z / D=0714)$, where the strong interaction plume-thermosiphon required the apparition of the two rotating rolls. A second zone $(0714<\mathrm{z} / \mathrm{D}=2.14)$, where develops the interaction flow. A last zone (z/D > 2.14), where turbulence is fully developed, occupying the upper part of the channel.

The analysis of the temperature spectra shows a change in the vortex size as one move away from the plume source. Indeed, the big vortex energy carriers, dependent on entry conditions, and undergo stretching in the flow upward and give rise to vortex structures averages. During their growth they produce vortex of smaller size. This development accompanied by a transfer of energy toward more and more small vortex until the viscous forces dominate the flow.

The spectra of temperature fluctuations obtained allowed to identify three distinct spectral regions that correspond to the mechanisms of energy transfer: a region of production due to turbulent flow, a region characteristic of the buoyancy anisotropic turbulence caused by buoyancy that modifies inertial range of balance in which energy of large vortex is transferred to smaller scales and finally, a dissipation region where viscous forces are predominant. This behavior follows the cascade energy scenario.

This study also shows that the proportion of energy corresponding to the small (dissipative) in the partially enclosed environment is more important than in the unlimited environment characterized by large vortex, energy carriers. The formation of these dissipative turbulent structures quickly homogenized fluid at the system exit.

\section{REFERENCES}

Abdelhakim, B., D. René and F. Jean Raymond (2007). An experimental study of the thermal plume developed above a finite cylindrical heat source to validate the point source model. Experimental Thermal and Fluid Science 31, 649-659.

Agator, J.M. and S. Doan Kim (1982). Turbulent structure of axisymmetric thermal plumes. Mechanics Research Communications 9, 159-164.

Agator, J.M. (1983). Contribution à l'étude de la Structure Turbulente d'un Panache Thermique à Symétrie Axiale. Interaction du panache avec son environnement limité, Thèse, Université de Poitiers.

Bouslimi, J. and L. Dehmani (2005). Experimental investigation of the thermal field of a turbulent plume guided by a cylinder- preliminary results. Experimental Thermal and Fluid Science 29, 477484.

Brahimi, M., L. Dehmani and S. Doan Kim (1989). Structure turbulente de l'écoulement d'interaction de deux panaches thermiques. Int. J. Heat Mass Transfer 32, 1551-1559.

Brahimi, M., M. Lamour and S. Doan Kim (1988). Champs moyens et fluctuants des panaches thermiques isolés ou en interaction. Rev. Gen. Therm. 315-316, 236-243. 
Brahimi, M. and S. Doan Kim (1986). Experimental and numerical predictions of the mean flow of a turbulent pure plume. Arch. Mech. Warszawa 38, 519-528.

Corrsin, S. (1951). On the spectrum of isotropic temperature fluctuations in an isotropic turbulence. J. Appl. Phys. 22, 469-473.

Dehmani, L., S. Doan Kim and L. Ghaboue (1996a). Turbulent structure of an axisymmetric plume penetrating a strong density stratification. Int. J. Heat Fluid Flow 17, 452-459.

Dehmani, L., S. Doan Kim, L. Gbahoue and F.X. Ronge 're (1996b). Influence of a strong density stratification axisymmetric plume. Exp. Fluids 21, 170-180.

Dai, Z., Tseng, L.K., Faeth, G.M., 1994, Structure of round, fully developed, buoyant turbulent plumes, J. Heat Transfer 116, 409-417.

Dai, Z., L.K. Tseng and G.M. Faeth (1995). Velocity statistics of round, fully developed, buoyant turbulent plumes. J. Heat Transfer 117, 138-145.

Doan Kim, S. (1977). Contribution à l'étude de la Zone de Transition et de la Zone de Turbulence établie dans un écoulement de Convection Naturelle sur une Plaque Plane Verticale Isotherme. Thèse de Doctorat d'Etat, Université de Poitiers.

Elicer-Cortés, J.C., R. Contreras, D. Boyer, M. Pavageau and R.H. Hernández (2004). Temperature spectra from a turbulent thermal plume by ultrasound scattering. Experimental Thermal and Fluid Science 28, 803-813.

Elicer-Cortés, J.C., Fuentes, J.A. Valencia and C. Baudet (2000). Experimental study of transition to turbulence of a round thermal plume by ultrasound scattering. Experimental Thermal and Fluid Science 20, 137-149.

Elicer-Cortés, J.C., A. Navia, D. Boyer, M. Pavageau and R.H. Hernández (2006). Experimental determination of preferred instability modes in a mechanically excited thermal plume by ultrasound scattering. Experimental Thermal and Fluid Science 30, 355-365.

Fukui, K., M. Nakajima and H. Ueda (1991). Coherent structure of turbulent longitudinal vortices in unstably-stratified turbulent flow. International Journal of Heat and Mass Transfer 34, 2373-2385.

Fulachier, L. and R.A. Antonia (1984). Spectral analogy between temperature and velocity fluctuations in several turbulent flows. International Journal of Heat and Mass Transfer 27, 987-997.

Jean, B.F. (1822). La Transformée de Fourier (Internet).
Kotsovinos, N. (1991). Turbulence spectra in free convection flow. Phys. Fluids A 3(1), 163 -167.

Lisin, F.N. and G. Hetsroni (1995). Spectrum of temperature fluctuations in high temperature turbulent gas-particle flow. International Journal of Heat and Mass Transfer 38,723-730.

Lumley, J.L. (1965). The spectrum of nearly inertial turbulence in a stably stratified fluid. J. Atmos. Sci. 21(1), 99-102.

Minh, V.P., P. Frédéric and S. Doan Kim (2006). Effect of swirl on pure turbulent thermal plume development. International Journal of Heat and Fluid Flow 27, 502-513.

Mahmoud, A.O.M., R. Ben Maad and A. Belghith (1998). Interaction d'un écoulement de thermosiphon avec un panache thermique à symétrie axiale : étude expérimentale. Rev. Gén. Therm. 37, 385-396.

Noto, K. and H. Okamoto (1991). Thermal plume from a heat source in thermally stable stratified air: Fundamental characteristics of heat island phenomenon. Energy and Buildings 15, 183-190.

Pera, L. and B. Gebhart (1975). Laminar plume interactions. J. Fluid Mech. 68, 259-271.

Cooley, J.W. and J.W. Tukey (1965). An Algorithm for the Machine Calculation of Complex Fourier Series. Math. Comput 19, 297-301.

Roland, S., Préface de Brian and E. Launder (1993). Modélisation et Simulation des écoulements Turbulents. Hermès, Paris.

Shabbir, A. and W.K. George (1994). Experiments on round turbulent buoyant plume. J. Fluid Mech. $275,1-32$.

Sangras, R., Z. Dai and G.M. Faeth (1998). Mixing structure of plane self-preserving buoyant turbulent plumes. ASME J. Heat Transfer 120, 1033-1041.

Takeo, S.S. and Y. Noboru (2004). Experimental and numerical investigation of thermal plume in urban surface layer. Experimental Thermal and Fluid Science 28, 585-595.

Zinoubi, J. (2003). Etude de l'interaction d'un écoulement de Thermosiphon avec un Panache Thermique à Symétrie Axiale: Influence de Paramètre de Forme. Thèse de Doctorat, Université El-Manar, Faculté des Sciences de Tunis.

Zinoubi, J., R. Ben Maad and A. Belghith (2004). Influence of the vertical source-cylinder spacing on the interaction of thermal plume with a thermosiphon flow: an experimental study. Exp Therm. Fluid. Sci. 28, 329-336. 